\title{
SUSTAINABILITY ANALYSIS OF REUSED INDUSTRIAL BUILDINGS IN CHINA: AN ASSESSMENT METHOD
}

\author{
Wei TIAN ${ }^{1^{*}}$, Xingju ZHONG ${ }^{1}$, Guangmin $Z \mathrm{ZHANG}^{1}$, Yang Miang $\mathrm{GOH}^{2}$ \\ ${ }^{1}$ School of Civil Engineering, Xian University of Architecture and Technology, Xian 710055, China \\ ${ }^{2}$ School of Design and Environment, National University of Singapore, Singapore 117566, Singapore
}

Received 10 May 2020; accepted 15 January 2021

\begin{abstract}
The sustainable development of old industrial buildings is in line with the national construction strategy and has an important impact on current urban renewal. Only by achieving a unified balance among economic, social, and environmental factors can reused industrial buildings be considered sustainable. However, there are no relevant sustainability assessment indicators and methods for reused industrial buildings in China. The purpose of this study was to provide a reasonable and effective method for assessing the sustainability of reused industrial buildings. First, this study analysed the factors influencing reused industrial building sustainability through a project investigation. Second, based on the assessment indicator setting procedure, the sustainability assessment indicator system for reused industrial buildings was optimised. Moreover, a multi-level sustainability assessment model based on extenics was established to identify the correlation functions of indicators with different attributes. Finally, a case was considered to verify this assessment method. The results showed that this assessment method in good agreement with the actual state of the case was validated to be more effective and practical. The assessment method could provide a basis for decision-making to improve sustainability and could be adopted by relevant rating agencies to determine the sustainability level of reused industrial buildings.
\end{abstract}

Keywords: old industrial buildings, reuse, sustainability assessment, extenics.

\section{Introduction}

In the 1990s, major cities in China underwent industrial restructuring, and a large number of old industrial buildings were demolished. However, in recent years, China has attached increasing importance to inheriting urban history and culture and protecting the ecological environment. In particular, the government outlined specific construction strategies for an ecological civilisation society. Therefore, the sustainable, recyclable, green, and energy-saving mode will become the main trend of urban construction in the future, and the reuse of old industrial buildings, this term derived from relevant industry standards in Mainland China similar to the adaptive reuse of industrial buildings in Hong Kong (Tan et al., 2018a) and the regeneration of industrial facilities in South Korea (Eom \& An, 2018), conforms to the current policy and development trend.

As it has gradually matured, the idea of sustainable development has progressively been accepted in the construction industry (Turcu, 2013). It is recognised that the development and application of building sustainability assessment methods can better solve the problem of sustain- able construction. Scientific and reasonable sustainability assessment can guide project plan optimisation, planning and design, construction process control, and management thought adjustment. Some reuse projects have proved that sustainability assessment can greatly promote the realisation of various construction goals.

Many developed countries have studied and established sustainability-based assessment systems for buildings, considering their construction characteristics (Zhang et al., 2014). The relatively complete and mature green building assessment systems include LEED, BREEAM, CASBEE, GBC, NABERS, Eco Profile, and ESCALE (Ali \& Al Nsairat, 2009; Drejeris \& Kavolynas, 2014). China is relatively late in researching assessment systems for green buildings. In 2004, it implemented the Assessment System for Green Building of the Beijing Olympic (Qin et al., 2017). In 2006, it implemented the Assessment Standard for Green Building (ASGB), which was revised in 2014 and 2019. Although some of the assessment indicators in the ASGB could be used in assessing the reuse of old

${ }^{*}$ Corresponding author. E-mail: tianwei@xauat.edu.cn

Copyright $\odot 2021$ The Author(s). Published by Vilnius Gediminas Technical University

This is an Open Access article distributed under the terms of the Creative Commons Attribution License (http://creativecommons.org/licenses/by/4.0/), which permits unrestricted use, distribution, and reproduction in any medium, provided the original author and source are credited. 
industrial buildings as civil buildings, others are not appropriate for this application and diverge from reality. Therefore, China and other countries lack a complete, systematic, and applicable sustainability assessment system for reused industrial buildings.

Although more green building assessment standards have emerged, research on building sustainability assessment is ongoing. Hannouf and Assefa (2018) proposed an LCSA-based decision-making analysis framework for sustainable development; analysed the interrelationships among the three dimensions of environment, economy, and society; and provided sustainable development solutions. Tokbolat et al. (2018) analysed the existing residential building types in Kazakhstan and assessed the gap between the existing buildings and green buildings specified in the standards. Vucicevic et al. (2013) analysed the factors influencing the sustainability assessment (economy, environment, and society) by investigating an existing residential area in Serbia and developed a sustainability assessment indicator framework for residential buildings. Zhang et al. (2016) selected the old industrial zone in Tiexi as a case study to assess the overall sustainable performance of an old industrial zone after brownfield restoration by observing the changes in the input-output flow. This multi-standard assessment method is recognised to a certain extent.

The reuse of old industrial buildings has been increasing in China for more than 20 years, and various aspects of corresponding research have also emerged; however, research on the sustainability of reused industrial buildings is scarce and lacking. Our team has been committed to researching the reuse of old industrial buildings and has compiled several specifications for related industries. The sustainability assessment of the reuse of old industrial buildings is a new research focus in China. Fan et al. (2013) summarised the key indicators, preconditions, and guaranty factors for the reuse potential of old industrial buildings and estimated the future value added to the project through a comprehensive assessment of the reuse potential; this was a pre-project assessment method and did not involve the project cost, construction uncertainty, and other factors. In similar studies, some researchers have focussed on the factor analysis of reused urban industrial buildings and old neighbourhood regeneration. Chan et al. (2015a) obtained information on a revitalisation scheme from questionnaires and analysed the impacts of revitalising industrial buildings from economic, social, and cultural perspectives. Tan et al. (2018a) examined industrial building reuse in Hong Kong and found 33 factors that affected their adaptive reuse. Almeida et al. (2018) compared different sustainability assessment systems to find suitable sustainability indicators for the rehabilitation in old urban centres in Portugal. By analysing urban rehabilitation and regeneration policies, they defined the assessment criteria and created a simplified method model, which was considered a useful and easy-to-use tool by all the actors.

Subjective assessment methods such as expert scoring, Delphi, and analytic hierarchy processes are commonly applied in sustainability assessment, which quantifies indicators based on a high level of expert knowledge (Lee
\& Lim, 2018). If an artificial neural network is applied, a function approximation of the nonlinear relationship between the input and output data can be realised to reduce the influence of subjective factors (Zhang et al., 2018). However, structured data are much more difficult to obtain, which makes it difficult to satisfy the conditions for applying this method. Based on the indicator data collected in a survey, TOPSIS can be used to assess the sustainability of projects involving the reuse of old industrial buildings (Peng et al., 2019), but the TOPSIS assessment method cannot further analyse the remodelling of reused industrial buildings.

Because of economic, cultural, and environmental differences in different regions of China, the uncertainties affecting the implementation of projects are complex and numerous (Li et al., 2013). Because of the lack of original data on most old industrial buildings and the limited number of reused industrial buildings that have been in good operating condition, it is difficult to conduct quantitative analyses of linguistic variables in the assessment indicators. Meanwhile, the quantification of linguistic variables is different from metric variables and their values may come from different types of sets. Therefore, considering the current situation, this study selected the extenics method to analyse the sustainability of reused industrial buildings. The extenics theory is an interdisciplinary subject involving systematic science, thought science, and mathematics. Based on the matter element and extension set (including fuzzy set and classical set) concepts, the relationship and transformation of quality and quantity can be considered simultaneously, which is mainly used to solve the problem of incompatibility (Liu et al., 2019). Thus, this method can eliminate the incompatibility between subjective and objective indicators and output reasonable results. The extension set explores the degrees that research objects possess certain characteristics and transformations from a transformable perspective. It is used to study changing classifications, along with the classified changes and transformation of contradictory problems (Gu et al., 2019). It is an emerging design methodology for addressing problems with conflicting, irreconcilable design requirements. Extenics has a solid foundation set for formalising the descriptions of matter, information, knowledge, and their relations with the real world with which they engage.

This study holds that the sustainability assessment of reused industrial buildings is a multi-level assessment process that still follows the previous practise, classifies and weights indicators, and forms a multi-angle and multiindicator sustainability assessment indicator system.

\section{Methods}

This study initially utilised sustainability assessment indicators to assess the sustainable development status of China's reused industrial buildings to establish a sustainability assessment model. Based on a review of the literature on the application of assessment methods and indicators, the research process used in this study is shown in Figure 1. 


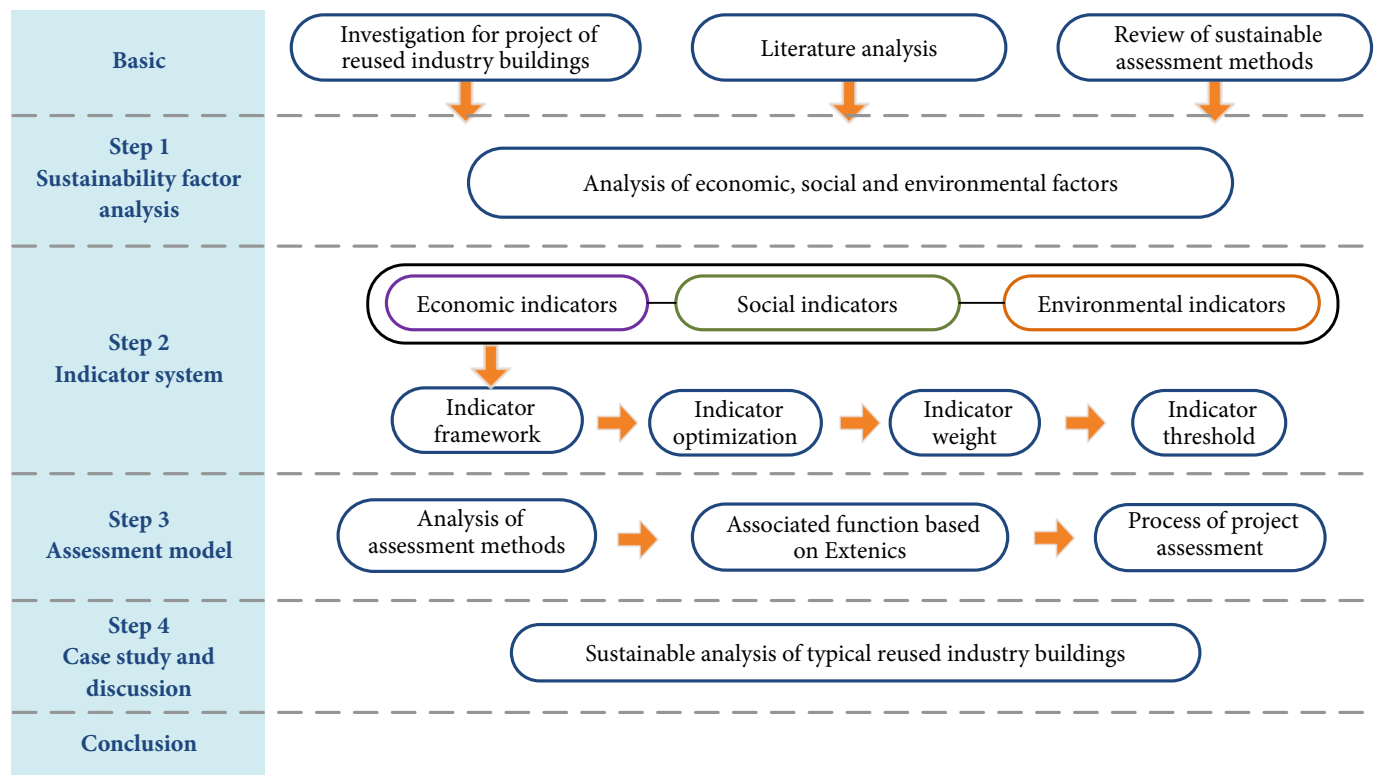

Figure 1. Sustainability analysis process for reused industrial buildings

Step 1: Analyse the factors influencing project sustainability from three aspects: economy, society, and environment, and set the tone for the selection of follow-up indicators.

Step 2: First, through a literature review and project surveys, the sustainability assessment indicator framework (economic indicators, social indicators, and environmental indicators) is initially established. Second, importance and correlation analyses are used to optimise the indicators. Considering that subjective and objective assessment indicators may exist simultaneously, the indicator weights are determined using a combination weighting method. Finally, the corresponding literature and national standards are used to set up the assessment indicator thresholds and complete the establishment of the assessment indicator system.

Step 3: Compare and analyse the relevant assessment methods, select a method suitable for sustainability assessment, and set the assessment process according to the characteristics of the selected method.

Step 4: Select a typical reuse project involving old industrial buildings and employ the established assessment model to analyse its sustainability.

\section{Sustainability indicators for reused industrial buildings}

\subsection{Surveys on reused industrial buildings}

Since 2009, our research group has conducted several surveys on reuse projects involving old industrial buildings in China and collected 148 cases in 30 cities. In this research process, through on-site investigations and questionnaires, related government personnel, project investors, designers, users, and late operators were consulted to collect historical data, project plans, reuse design drawings, and building entity images related to the reuse case, to fully understand the reuse background and reuse status of the project and to clarify the cause, purpose, and existing problems of reused industrial buildings. The on-site information and survey results that were preliminarily collated to form a basic database for reused industrial buildings provide a basis for analysing the factors influencing the sustainability of reused industrial buildings. The research process obtained some basic information, and the relevant cities and numbers of reused industrial buildings are shown in Figure 2.

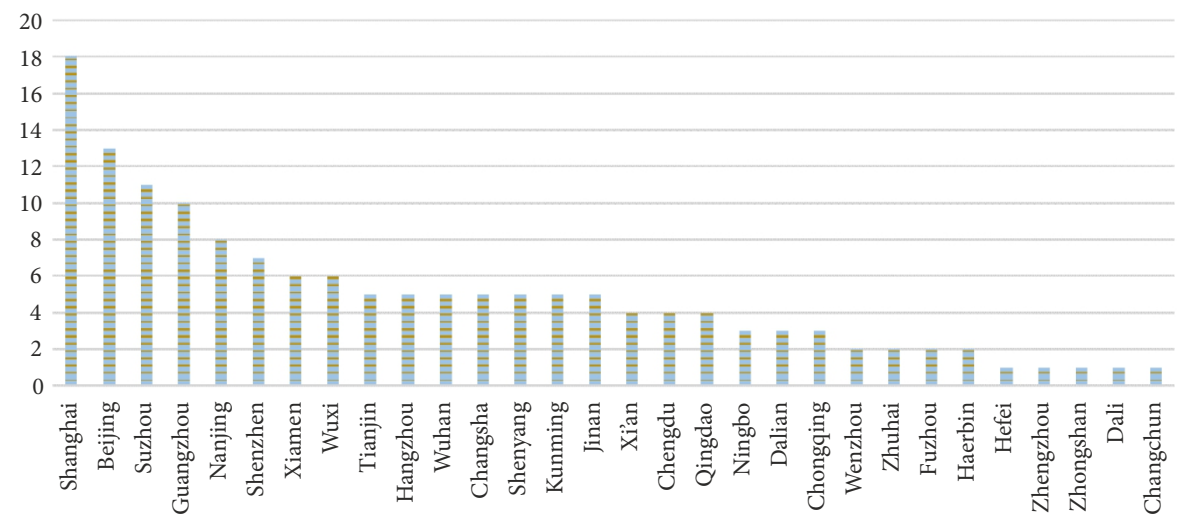

Figure 2. Distribution of reused industrial buildings in China 
As a result of China's industrial development process, the construction years for China's old industrial buildings are unevenly distributed, with the structure types also changing with the construction years. The distributions of the construction years and structure types are shown in Figures 3 and 4, respectively.

The reused industrial buildings have several characteristics. 1) Most of them were built in the early part of the period considered and have rich historical and cultural connotations. 2) Most of the building structures are intact, and more than $90 \%$ of the structures are masonryconcrete or reinforced concrete with good bearing capacities. 3) The reuse of these early buildings requires a large amount of work, and the reconstruction technology is relatively complex. Measures such as heritage protection have further increased the cost of reuse. 4) Because of the unique attraction of its location and cultural characteristics, a reused industrial building has commercial and cultural attributes, which enhance its economic value.

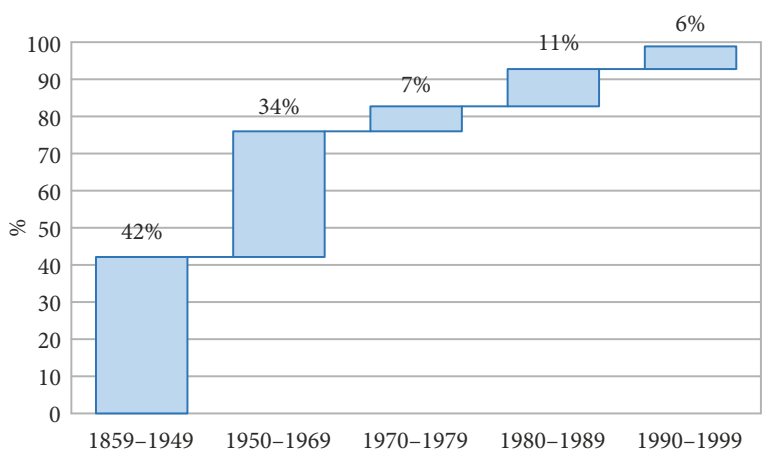

Figure 3. Construction years of reused industrial buildings

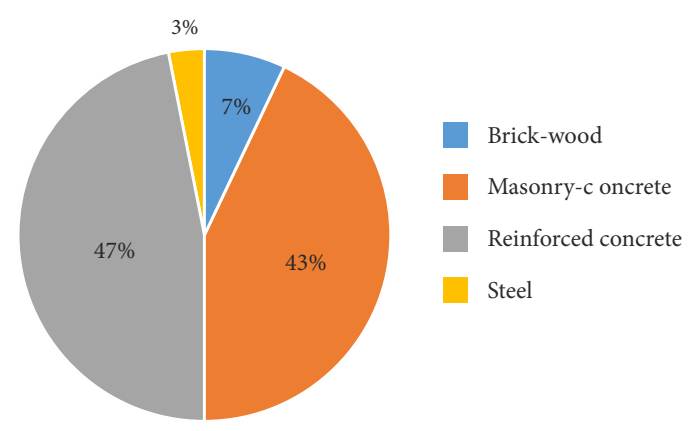

Figure 4. Structure types of reused industrial buildings

\subsection{Indicator selection procedures}

Indicators are used to measure the essential attributes and characteristics of the assessment objects from a quantitative or qualitative perspective. The indicator system refers to a set of indicators that are relatively independent of each other. A reasonable and effective indicator system is a prerequisite for the sustainability assessment of reused industrial buildings and is directly related to the rationality of the assessment results (Nilashi et al., 2015). Therefore, the selection of indicators is a key link in the assessment process.

The sustainability assessment of reused industrial buildings belongs to the post-assessment category. Its main function is to determine whether the economic, social, and environmental impacts of a project in the process of planning, construction, and operation have reached the expected goals; obtain experience from the problems found, provide effective reference information for future project decision-making; and improve the level of decision-making. Based on the theory of sustainable development, the indicator system for the sustainability assessment of reused industrial buildings is a set of indicators that need to comprehensively, accurately, and effectively reflect the decision-design-construction-operation rationality during the entire process for the project.

The reuse of old industrial buildings commenced late in China, and a corresponding assessment system has not yet been developed. Different regions have different economic, cultural, and geographical environments, and there is a lack of effective historical data for reference (Li et al., 2018; Fan et al., 2013). Therefore, it is necessary to select applicable indicators through special investigations, and finally develop an assessment indicator system for reused industrial buildings. The entire process of selecting indicators is shown in Figure 5.

\subsection{Preliminary assessment indicators}

The concept of 'low-carbon and green buildings' has become deeply rooted in the construction industry. However, because of the unbalanced regional development in China, building reuse based on the concept of sustainable development is still limited to some extent, and there is no unified and effective measurement standard to comprehensively assess the level of reuse for industrial

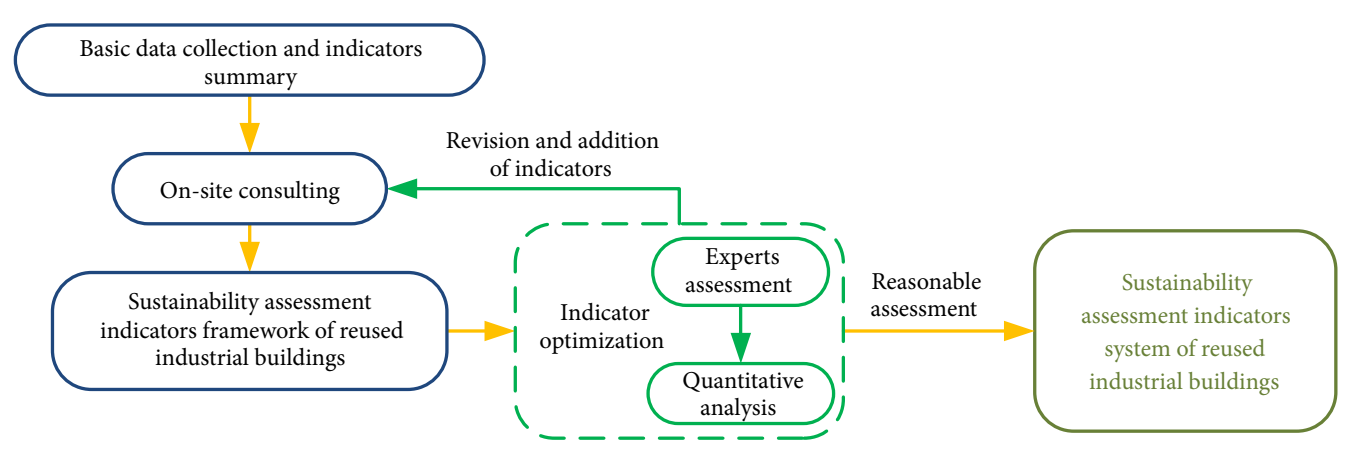

Figure 5. Sustainability assessment indicator selection procedures for reused industrial buildings 
buildings. To break through the 'bottleneck' in the reuse of old industrial buildings, it is necessary to start from the perspective of sustainable development; seek a unified balance between economic, social, and environmental aspects; and establish feasible assessment criteria. Sustainability assessment indicators for reused industrial buildings were selected from ASGB, the building sustainability indicators of the related literature, and surveys related to reused industrial buildings. These indicators were divided into three types: economy, society, and environment.

\section{Economy}

During the investigation, it was found that in different reuse projects, there were large differences in the amounts of investment per unit area, rates of return, etc. For example, the unit area investment for the Beijing 768 Creative Industry Park project was 291.12 yuan $/ \mathrm{m}^{2}$, while the unit area investment for the Tianjin No. 6 Creative Industry Park project was 1724.14 yuan $/ \mathrm{m}^{2}$. At the same time, there were also some cases where the investment per unit area of the reuse project far exceeded the cost for local new construction of the same type.

For investors, a general project without sufficient benefit cannot be carried out. The economic benefit of a project is generally determined using a financial assessment (Ameen \& Mourshed, 2019) (public or government-led projects need to be assessed in relation to the national economy). Commonly used quantitative economic assessment indicators mainly include the reuse cost (Ameen \& Mourshed, 2019), dynamic payback period (Banani et al., 2016), internal return on investment (ROI) (Mahmoud et al., 2019), rate of return (IRR), and net present value rate (NPVR) (Almeida et al., 2018). Some reuse projects have had complex and diverse sources of investment, which have led to uncertainty about the financial risks. The survey found that the financial risks, investment plans, and financing models had significant impacts on the project benefits. For reused industrial buildings, the complexity and applicability of reuse technology directly affect the cost of reuse (Section 2.1). Therefore, the economic efficiency of the reuse techniques (Tan et al., 2018b) should be taken into consideration.

\section{Society}

If construction projects conflict with the wishes of residents, the expected economic benefits to the investors will not be realised. In contrast, if the social response to construction projects is exceptionally good, it is bound to improve the economic benefits to the investors. It can be seen that the economic benefits and social benefits are positively correlated. Government-led reuse projects tend to focus more on their social impacts.

The indicators that measure a project's social benefits are commonly qualitative. For a reused industrial building project, these mainly include the impact on neighbourhood development (Fan et al., 2013), capacity for im- proving public health (Atanda, 2019), coordination with neighbours (Si et al., 2016), ability to provide employment (Ameen \& Mourshed, 2019), ability to provide services (Tupenaite et al., 2018), disturbance to residents (Eom \& An, 2018), and civilised construction (Ameen \& Mourshed, 2019). Surveys on reused industrial buildings have been conducted for more than 10 years. In later research, it was found that culture is also an important component of the sustainability of reused industrial buildings (Chan et al., 2015b). Because of insufficient data samples related to culture, only some cultural indicators with prominent social influence were considered in social factors, such as the coordination with culture, protection of cultural relics (Tan et al. 2018b), and promotion of a regional image (Chan et al., 2015c).

\section{Environment}

As the need to establish an environmentally friendly society has gradually been recognised, the promulgation of the ASGB shows that reuse projects have begun to be included in the green construction period. Especially in the ASGB, some indicators can also be used for the assessment of reused industrial building projects, including the indoor environment quality (Saraiva et al., 2018), air pollution, noise pollution, and green building management (Mahmoud et al., 2019).

Some relevant researchers have pointed out that an environmental sustainability assessment should integrate the environment (Fan et al., 2013), environmental sensitivity (Si et al., 2016), rational use of land (Zhang et al., 2014), sewage disposal (Tan et al., 2018b), and use of renewable energy (Banani et al., 2016). The survey found that when reusing old industrial buildings, many experts and project managers mentioned the use of recyclable materials, the reduction of energy consumption, waste disposal, and special pollution disposal, which are closely related to the environmental sustainability of reused industrial buildings.

In addition, some researchers discussed sustainability evaluation dimensions related to laws and policies (Chan et al., 2015a, 2015b, 2015c; Tan et al., 2018b). However, in the early investigation, it was found that there were few laws or policies related to the reuse of old industrial buildings in Mainland China. Only in recent years, a few cities have issued some related policies, which are still not comprehensive, and national laws or policies have not been formulated. Hence, there is not enough data or information to discuss the impact of laws or policies on the sustainability of reused industrial buildings. Nevertheless, the proposed sustainability assessment indicators and model could promote the formulation of relevant laws or policies to a certain extent.

Considering the actual development of old industrial building reuse in China, a preliminary framework of the sustainability assessment indicators for reused industrial buildings (Figure 6) can be summarised from the three aspects of the economy, society, and environment. 


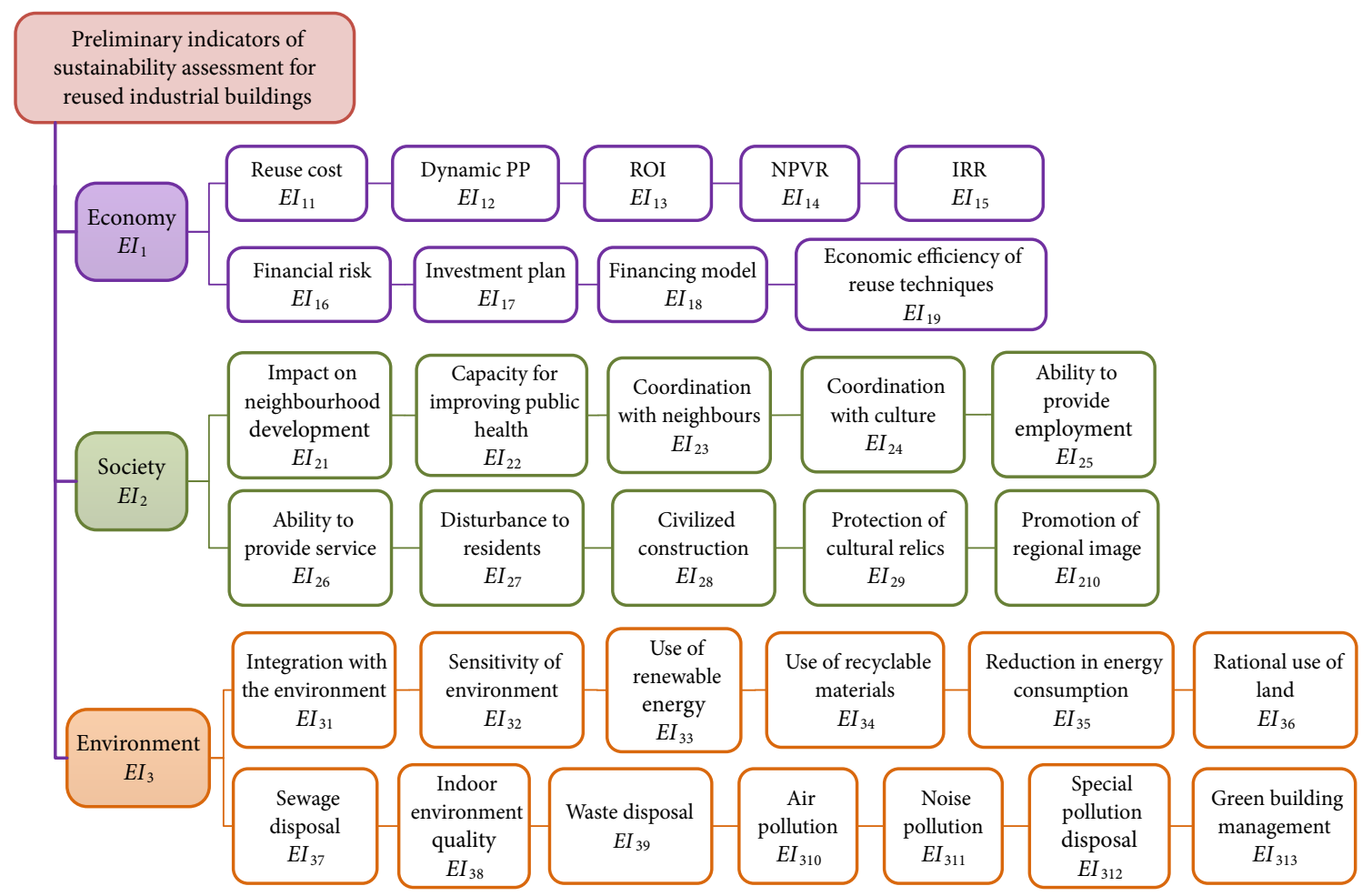

Figure 6. Preliminary indicators of sustainability assessment for reused industrial buildings

\subsection{Optimization of assessment indicators}

During the indicator selection process, a total of 56 valid questionnaires were recovered, of which 26 were provided by government personnel (responsible persons in departments such as the planning bureau, land bureau, and commission of economy and information technology), 18 were provided by people engaged in the design of old industrial buildings in various regions, and 12 were provided by researchers who have been engaged in this field for a long time.

Based on the preliminary indicators shown in Figure 6 , the optimal sustainability assessment indicator system for reused industrial buildings was developed through repeated exchanges and demonstrations with experts (Delphi method) and analyses of the importance and dispersion degree of the indicators.

A questionnaire was specially created to analyse the importance and dispersion degree of the indicators. In this questionnaire, the interviewed experts made judgements on the importance of the three categories of economic, social, and environmental indicators, which were divided into five levels: very unimportant -1 , unimportant -2 , average -3 , important -4 , and very important -5 . In this study, the objective degree of the questionnaire source was the same, and the data obtained from the survey were equally weighted.

\section{Indicator importance analysis method}

The analysis of the importance of the indicators was based on the comparison of the importance indexes, $R I I_{i}$, where a relatively small index indicated that the experts agreed that this indicator was relatively minor compared with other indicators, and could be properly eliminated according to the actual assessment (Li et al., 2013).

$$
R I I_{i}=100 \times \frac{N_{i 1} \times 1+N_{i 2} \times 2+N_{i 3} \times 3+N_{i 4} \times 4+N_{i 5} \times 5}{5 N},
$$

where $i=1,2,3, \ldots, m$, and $N_{i 1}-N_{i 5}$ represent the number of respondents, where indicator $i$ is assigned a number from 1 to 5 in the questionnaire, and $N$ is the total number of questionnaires.

\section{Indicator dispersion analysis method}

The dispersion degree of the indicator was mainly analysed based on variation coefficient $\delta_{i}$ calculated from the statistical data. When $\delta_{i}$ had a larger value, the experts' opinions on the indicator had greater divergence. Generally, the dispersion of the indicator in the later assessment was also relatively large, and the data were not reliable (Li et al., 2013). In this case, this indicator needed to be eliminated.

$$
\delta_{i}=\frac{\sigma_{i}}{\mu_{i}}, \mu_{i}=\frac{1}{n} \sum_{j=1}^{n} x_{i j}, \sigma_{i}=\sqrt{\frac{1}{n-1} \sum_{j=1}^{n}\left(x_{i j}-\mu_{i}\right)^{2}} .
$$

Through calculation, it can be seen that $E I_{13}, E I_{14}$, and $E I_{16}$ in the economic indicators; $E I_{24}$ and $E I_{28}$ in the social indicators; and $E I_{32}$ in the environmental indicators all belong to the indicators with the smaller importance indexes $\left(R I I_{i}<80\right)$. Moreover, the variation coefficients of these six indicators are larger $\left(\delta_{i}>0.25\right)$, indicating that the experts questioned showed great differences in the importance 
that they placed on these indicators. Therefore, these indicators were removed from the preliminary framework, and the rearranged indicators were collected and fed back to 56 interviewed experts. These were recognised by the experts, and finally formed the sustainability assessment indicators for reused industrial buildings (Table 1).

\subsection{Sustainability indicator weight}

Weight determination methods include subjective and objective methods. A subjective method weights each indicator according to the subjective importance assigned by decision-makers (Tupenaite et al., 2017), such as through an expert survey and analytic hierarchy process (AHP). An objective method mainly determines the weight according to the correlation and variation degree of the assessment indicator itself (Rani et al., 2014), such as through the entropy method and principal component analysis. A subjective method relies excessively on the subjective judgements of decision-makers and is susceptible to subjective factors, whereas an objective method avoids human factors but is affected by the random error of indicator samples (Tan et al., 2014). Comprehensive weighting can account for both subjective and objective weight information, which allows it to not only make full use of objective data but also meet the subjective wishes of decisionmakers (Wang et al., 2017). Therefore, the sustainability assessment of reused industrial buildings adopts a combination method that uses the minimum deviation sum (Lagrange condition extreme value principle) to determine the weight.

Sustainability assessment indicators are evaluated in two forms. Indicators such as $S I_{14}, S I_{15}, S I_{16}, S I_{32}, S I_{33}$, $S I_{39}$, and $S I_{310}$ are all evaluated based on quantitative values. At the same time, the other qualitative indicators with fuzzy attributes can be expressed using five linguistic variables (degrees of performance). Based on the literature review and expert consultation, these five linguistic variables were low, relatively low, middle, relatively high, and high, which corresponded to the five intervals of $[0,0.2)$, $[0.2,0.4),[0.4,0.6),[0.6,0.8)$, and $[0.8,1.0]$, respectively.

In this study, AHP was used to obtain the subjective weights: $w^{\prime}=\left(w_{1}^{\prime}, w_{2}^{\prime}, \ldots, w_{n}^{\prime}\right), w_{j}^{\prime} \geq 0$, and $\sum_{j=1}^{m} w_{j}^{\prime}=1$ (Wang et al., 2017). Based on Saaty's suggestion, the elements between 5 and $9(7 \pm 2)$ should be the ideal at one level (Mu \& Pereyra-Rojas, 2017). There are 12 indicators in the environmental category. Therefore, we grouped $S_{36}$, $S I_{38}$, and $S I_{311}$ into one temporary indicator 'pollution disposal', grouped $S I_{39}$ and $S I_{310}$ into 'pollution', and applied the AHP to acquire weights at this level. Subsequently, the original indicators such as $S_{36}, S I_{38}$, and $S_{311}$ were taken as the sub-level elements of their corresponding temporary indicator, and the AHP is applied a second time to obtain their respective weights. An entropy method was used to determine the objective weights, $w^{\prime \prime}=\left(w_{1}^{\prime \prime}, w_{2}^{\prime \prime}, \ldots, w_{n}^{\prime \prime}\right)$, of the indicators (Polat, 2016), and 13 projects involving reused industrial buildings with complete data were selected from 153 projects to determine the entropy weights.

The comprehensive weights $w$ of the indicators can be expressed as

$$
w_{j}=\alpha^{*} w_{j}^{\prime}+\beta^{*} w_{j}^{\prime \prime}
$$

where $\alpha^{*}$ and $\beta^{*}$ are the degrees of importance of $w^{\prime}$ and $w^{\prime \prime}$, respectively. Seeking the solution of $w$ is a constrained optimization problem that can be dealt with by the Lagrange multiplier method (Borwein \& Zhu, 2016). In this way, the values $\alpha^{*}$ and $\beta^{*}$ can be obtained as follows:

$$
\begin{aligned}
& \alpha^{*}=\frac{\sum_{i=1}^{m} \sum_{j=1}^{n} a_{i j} w_{j}^{\prime}}{\sum_{i=1}^{n} \sum_{j=1}^{m} a_{i j}\left(w_{j}^{\prime}+w_{j}^{\prime \prime}\right)}, \\
& \beta^{*}=\frac{\sum_{i=1}^{m} \sum_{j=1}^{n} a_{i j} w_{j}^{\prime \prime}}{\sum_{i=1}^{m} \sum_{j=1}^{n} a_{i j}\left(w_{j}^{\prime}+w_{j}^{\prime \prime}\right)},
\end{aligned}
$$

where $a_{i j}$ represents the standard value of the indicator $S I_{i j}$.

If an individual indicator was missing in the actual assessment process, the weight of the indicator could be evenly distributed among the other indicators in the same indicator layer. The final calculated weights are listed in Table 1 .

\section{Sustainability assessment model}

\subsection{Assessment method}

In the extenics theory, matter-element theory and extension mathematics are two theoretical bases of the objective world (Zhou et al., 2016). Matter-element theory attempts to reveal the combination between qualitative and quantitative research approaches, and extension mathematics quantifies certain contradictory problems with extension sets and corresponding correlation functions (He et al., 2016).

In the established indicator system, some indicators are assigned subjective data and others are assigned objective data. Thus, this study adopted extension prioritydegree assessment to analyse the sustainability of reused industrial buildings. The theoretical support of extension priority degree assessment is the extenics theory, which is a widely used engineering method in the extenics (Jiang et al., 2019). The basic process of the sustainability analysis based on extension priority-degree for reused industrial buildings is shown in Figure 7.

\section{Indicators}

The indicator set of an assessed object $Z$ is $S I=\left\{S I_{1}\right.$, $\left.S I_{2}, \cdots, S I_{n}\right\}$, which can be expressed as $S I=\left(c_{i}, V_{i}\right)$, with $c_{i}$ being a feature of an indicator and $V_{i}$ being the threshold as shown in Table 1. 
Table 1. Sustainability assessment indicator system of reused industrial buildings

\begin{tabular}{|c|c|c|c|c|c|c|c|c|c|}
\hline \multicolumn{6}{|c|}{ Sustainability assessment indicator system of reused industrial buildings } & \multicolumn{4}{|c|}{ Case data } \\
\hline $\begin{array}{l}\text { Category } \\
S_{i}\end{array}$ & $\begin{array}{c}\text { Weight } \\
\alpha_{i}\end{array}$ & $\begin{array}{l}\text { Indicators } \\
\qquad S I_{i j}\end{array}$ & $\begin{array}{c}\text { Range } \\
V_{i j}\end{array}$ & $\begin{array}{c}\text { Weight } \\
\alpha_{i j}\end{array}$ & $\begin{array}{c}\text { Correlation } \\
\text { function } \\
K_{i j}\end{array}$ & Score & $\begin{array}{c}\text { Correlation } \\
\text { degree }\end{array}$ & $\begin{array}{l}\text { Priority } \\
\text { Degree }\end{array}$ & $\begin{array}{l}\text { Comprehensive } \\
\text { priority degree }\end{array}$ \\
\hline \multirow{6}{*}{$\begin{array}{l}\text { Economy } \\
\left(S I_{1}\right)\end{array}$} & \multirow{6}{*}{0.3653} & Investment plan $S I_{11}$ & {$[0,1]$} & 0.1188 & $\mathrm{E}(12)$ & 0.82 & 0.82 & \multirow{6}{*}{0.6760} & \multirow{26}{*}{0.6532} \\
\hline & & Financing model $S I_{12}$ & {$[0,1]$} & 0.1022 & $\mathrm{E}(12)$ & 0.75 & 0.75 & & \\
\hline & & $\begin{array}{l}\text { Economic efficiency of } \\
\text { reuse techniques } S_{\mathrm{I} 1} 3\end{array}$ & {$[0,1]$} & 0.1823 & $\mathrm{E}(12)$ & 0.78 & 0.78 & & \\
\hline & & Reuse cost $S I_{14}$ & {$\left[a_{14}, b_{14}\right]$} & 0.2989 & $\mathrm{E}(10)$ & 1250 & 0.5 & & \\
\hline & & Dynamic PP $S I_{15}$ & {$\left[a_{15}, b_{15}\right]$} & 0.1211 & $\mathrm{E}(11)$ & 2.1 & 0.73 & & \\
\hline & & IRR $S I_{16}$ & {$\left[a_{15}, b_{15}\right]$} & 0.1766 & $\mathrm{E}(12)$ & 15.3 & 0.69 & & \\
\hline \multirow{8}{*}{$\begin{array}{l}\text { Society } \\
\left(\mathrm{SI}_{2}\right)\end{array}$} & \multirow{8}{*}{0.2142} & $\begin{array}{l}\text { Impact on } \\
\text { neighbourhood } \\
\text { development } S I_{21}\end{array}$ & {$[0,1]$} & 0.2704 & $\mathrm{E}(12)$ & 0.83 & 0.83 & \multirow{8}{*}{0.72} & \\
\hline & & $\begin{array}{l}\text { Capacity for improving } \\
\text { public health } S I_{22}\end{array}$ & {$[0,1]$} & 0.1085 & $\mathrm{E}(12)$ & 0.65 & 0.65 & & \\
\hline & & $\begin{array}{l}\text { Coordination with } \\
\text { neighbours } S_{23}\end{array}$ & {$[0,1]$} & 0.0810 & $\mathrm{E}(12)$ & 0.67 & 0.67 & & \\
\hline & & $\begin{array}{l}\text { Ability to provide } \\
\text { employment } S I_{24}\end{array}$ & {$[0,1]$} & 0.0901 & $\mathrm{E}(12)$ & 0.5 & 0.5 & & \\
\hline & & $\begin{array}{l}\text { Ability to provide } \\
\text { services } S I_{25}\end{array}$ & {$[0,1]$} & 0.0362 & $\mathrm{E}(12)$ & 0.45 & 0.45 & & \\
\hline & & $\begin{array}{l}\text { Disturbance to } \\
\text { residents } S I_{26}\end{array}$ & {$[0,1]$} & 0.0626 & $\mathrm{E}(11)$ & 0.25 & 0.75 & & \\
\hline & & $\begin{array}{l}\text { Protection of cultural } \\
\text { relics } S_{27}\end{array}$ & {$[0,1]$} & 0.2429 & $\mathrm{E}(12)$ & 0.75 & 0.75 & & \\
\hline & & $\begin{array}{l}\text { Promotion of regional } \\
\text { image } S I_{28}\end{array}$ & {$[0,1]$} & 0.1085 & $\mathrm{E}(12)$ & 0.74 & 0.74 & & \\
\hline \multirow{12}{*}{$\begin{array}{l}\text { Environment } \\
\left(\mathrm{SI}_{3}\right)\end{array}$} & \multirow{12}{*}{0.4205} & $\begin{array}{l}\text { Integration with the } \\
\text { environment } S I_{31}\end{array}$ & {$[0,1]$} & 0.0683 & $\mathrm{E}(12)$ & 0.68 & 0.68 & \multirow{12}{*}{0.5993} & \\
\hline & & $\begin{array}{l}\text { Use of renewable } \\
\text { energy } S_{32}\end{array}$ & {$[0.2,1]$} & 0.0310 & $\mathrm{E}(12)$ & 0.77 & 0.71 & & \\
\hline & & $\begin{array}{l}\text { Use of recyclable } \\
\text { materials } S I_{33}\end{array}$ & {$[0,0.5]$} & 0.1478 & $\mathrm{E}(12)$ & 0.34 & 0.67 & & \\
\hline & & $\begin{array}{l}\text { Reduction in energy } \\
\text { consumption } S_{34}\end{array}$ & {$[0,1]$} & 0.1463 & $\mathrm{E}(12)$ & 0 & 0 & & \\
\hline & & $\begin{array}{l}\text { Rational use of land } \\
S_{35}\end{array}$ & {$[0,1]$} & 0.1603 & $\mathrm{E}(12)$ & 0.74 & 0.74 & & \\
\hline & & Sewage disposal $S_{36}$ & {$[0,1]$} & 0.1002 & $\mathrm{E}(12)$ & 0.69 & 0.69 & & \\
\hline & & $\begin{array}{l}\text { Indoor environment } \\
\text { quality } S_{37}\end{array}$ & {$[0,1]$} & 0.0622 & $\mathrm{E}(12)$ & 0.73 & 0.73 & & \\
\hline & & Waste disposal $\mathrm{SI}_{38}$ & {$[0,1]$} & 0.0854 & $\mathrm{E}(12)$ & 0.69 & 0.69 & & \\
\hline & & Air pollution $S_{39}$ & {$[0,200]$} & 0.0493 & $\mathrm{E}(11)$ & 79 & 0.605 & & \\
\hline & & Noise pollution $S I_{310}$ & {$[30,65]$} & 0.0282 & $\mathrm{E}(11)$ & 35 & 0.857 & & \\
\hline & & $\begin{array}{l}\text { Special pollution } \\
\text { disposal } S I_{311}\end{array}$ & {$[0,1]$} & 0.0334 & $\mathrm{E}(12)$ & 0.67 & 0.67 & & \\
\hline & & $\begin{array}{l}\text { Green building } \\
\text { management } S_{312}\end{array}$ & {$[0,1]$} & 0.0876 & $\mathrm{E}(12)$ & 0.72 & 0.72 & & \\
\hline
\end{tabular}

\section{Matter-element}

Suppose a matter-element $M=(Z, C, V)$ is an ordered ternary for transformation. Specifically, the matter-element $M$ can also be written as:

$$
M=\left[\begin{array}{ccc}
Z & c_{1} & V_{1} \\
& c_{2} & V_{2} \\
& \vdots & \vdots \\
& c_{n} & V_{n}
\end{array}\right],
$$

where each sub-matter-element of $M$ is defined as $M_{i}=(Z$, $\left.C_{i}, V_{i}\right)$ for $i=1,2, \ldots, n$.

\section{Correlation function}

In the extenics, the correlation function to describe the degree of correlation of each indicator can be expressed as $K(z)$. Before priority-degree calculation, the obtained degrees of correlation should be normalized as: 


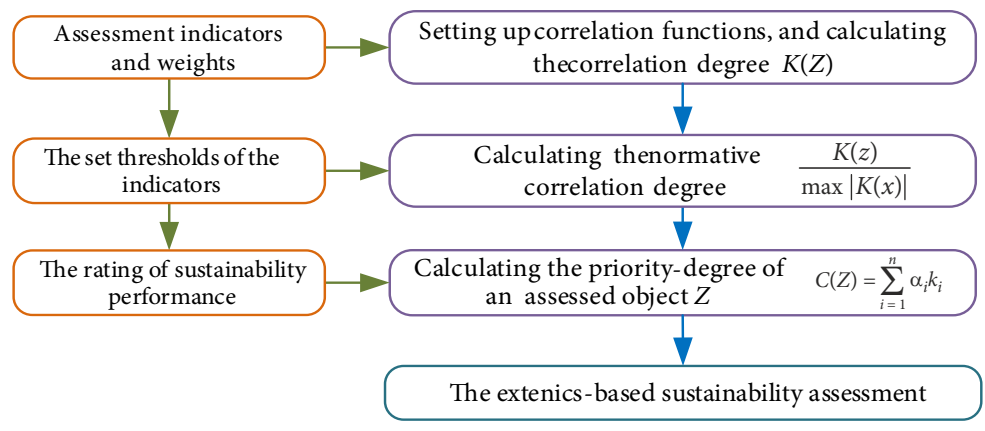

Figure 7. The extenics-based sustainability assessment

$$
k(x)=\frac{K(z)}{\max |K(x)|} .
$$

The calculation of the value of $k(x)$ is related to the threshold of the indicator, and the detailed calculation process is shown in Section 3.3.

\section{Priority-degree}

The priority-degree, the sum of the weighted correlation-degree of each indicator, indicates the sustainability performance level of an assessed object $Z$. The prioritydegree of the assessed object $Z$ can be defined as:

$$
C(Z)=\sum_{i=1}^{n} \alpha_{i} k_{i}
$$

The detailed calculation process of the priority-degree in the two cases will be introduced in Section 3.4.

\subsection{Setting assessment indicator range}

In determining the indicator range, priority should be given to the official planned standard thresholds of the relevant social, economic, and environmental indicators. In addition, the case data collected in the current investigation should be used as the basis to determine the value range. Considering the changing trends of the regional economy and environment, the estimated value in this changing process is used as the basis for determining the value range.

The indicator describing the degree is usually a kind of fuzzy measurement such as the 'investment plan $\left(S I_{11}\right)^{\text {' }}$ or 'financing model $\left(S I_{12}\right)$ ', in which case the value range is $[0,1]$, an assessment value of one is considered to indicate the largest correlation degree for the indicator range. However, for measurements such as the 'disturbance to residents $\left(\mathrm{SI}_{26}\right)$ ' and 'air pollution $\left(\mathrm{SI}_{39}\right)$ ', an assessment value of 0 indicates the largest correlation degree for the indicator range. It is easy to understand that a project with less disturbance and pollution is more sustainable.

Some indicators are greatly influenced by a region's society, economy, and environment, and there are regional differences. These make it difficult to set the value range, and it needs to be combined with an actual investigation. According to the principle of extension transformation, for example, the 'reuse cost $\left(S I_{14}\right)$ ' can be converted into the cost per unit square metre, and its value range can be expressed as $\left[a_{14}, b_{14}\right]$. Considering regional differences, the assessment of the value of $\left(a_{14}+b_{14}\right) / 2$ is set as the maximum correlation.

To improve the standardisation of the assessment process, some indicators need to be set by referring to national standards. For example, the ASGB has relevant provisions on the 'use of renewable energy $\left(\mathrm{SI}_{32}\right)$ '. Therefore, the value range of this indicator is set at $[0.2,1]$, and it is believed that the correlation degree is the largest when the assessment value is one.

The sustainability assessment indicator ranges of the reused industrial buildings were set based on the above analysis (Table 1).

\subsection{Establishment of correlation functions}

A correlation function is a quantitative tool used in extenics. In extension priority degree assessment, the degree of correlation between the assessment value of the indicator and its range is established through the correlation function to carry out the assessment (Lou et al., 2018). Based on the range attributes of the sustainability assessment indicators for reused industrial buildings, the correlation function $k(x)$ is selected as follows:

Let the interval of the quantitative range be

$$
\begin{aligned}
& X=[a, b], M \in X, \\
& k(x)=\left\{\begin{array}{l}
\frac{x-a}{M-a}, x \leq M \\
\frac{b-x}{b-M}, x \geq M
\end{array},\right.
\end{aligned}
$$

where $k(x)$ reaches its maximum value when $x=M$, and $k(M)=1 ; x \in X$, and $x \neq a, b \Leftrightarrow k(x)>0 ; x \notin X$, and $x \neq a, b \Leftrightarrow k(x)<0$; and $x=a$ or $x=b \Leftrightarrow k(x)=0$.

Different indicator ranges represent different attributes, and the maximum correlations of these different indicators are also different. Therefore, an appropriate correlation function should be selected according to the specific situation. Considering the three cases of maximum correlation degree: $M=(a+b) / 2, M=a$, and $M=$ $b$, the calculation formulae for the correlation degree of an assessment indicator are as follows, and the selected correlation functions are listed in Table 1. 


$$
\begin{aligned}
& M=(a+b) / 2, k(x)=\left\{\begin{array}{l}
\frac{2(x-a)}{b-a}, x \leq \frac{a+b}{2} \\
\frac{2(b-x)}{b-a}, x \geq \frac{a+b}{2}
\end{array}\right. \\
& M=a, k(x)=\left\{\begin{array}{c}
\frac{x-a}{b-a}, x<a \\
\frac{b-x}{b-a}, x>a \\
k(a)=0 \vee 1, x=a
\end{array}\right. \\
& M=b, k(x)=\left\{\begin{array}{c}
\frac{x-a}{b-a}, x<b \\
\frac{b-x}{b-a}, x>b \\
k(b)=0 \vee 1, x=b
\end{array}\right.
\end{aligned}
$$

After the correlation degree was calculated, the data collected from the 13 groups of surveys were used to calculate the normative correlation degree, and the priority degree could be calculated.

\subsection{Assessment process}

It is generally believed that when the comprehensive correlation degree of an assessment indicator is greater than zero, the assessed object $Z$ meets the requirements and has sustainability at this stage. The assessment process was established based on the basic principles of extension priority-degree assessment, as shown in Figure 8.

\section{Primary assessment}

The primary assessment is performed before the priority degree assessment, which is a characteristic of extension priority-degree assessment and more in line with the actual situation.

The primary assessment is used to determine whether the policies, regulations, and standards for the assessed object meet the mandatory requirements for social, economic, environmental, and structural safety in the process of project construction. In particular, if the reused industrial building to be assessed cannot meet the requirements for structural safety stipulated in the mandatory regulations, it is not sustainable.

If the assessed object meets the mandatory conditions, it is considered that the project can enter the link of priority degree assessment.

\section{Priority degree assessment}

After calculating the normative correlation degree of the assessment indicator, a judgement is made using the following formula:

$$
C_{1}\left(Z_{i j}\right)=\stackrel{3}{\wedge} \wedge_{i=1}^{n} k_{i j}
$$

where $\wedge$ means to take the minimum of all $k_{i j}$ values for $i \in(1,2,3)$, and $j \in(1,2, \cdots, n)$.

$$
\text { - } C_{1}\left(Z_{i j}\right)>0 \text {. }
$$

The assessed object passes the priority degree assessment as a whole. The fact that the correlation degree of all the indicators is greater than zero under limited circumstances indicates that the assessed object can also pass the comprehensive priority-degree assessment, which means that the assessed object is considered to be sustainable. If the sustainability of the reused industrial building needs to be further analysed, this can be achieved through a comprehensive priority degree assessment.

- $C_{1}\left(Z_{i j}\right) \leq 0$.

It is considered that the assessed object as a whole fails to pass the priority degree assessment. Thus, it is necessary to further determine whether the reused industrial building has sustainable development potential, and it should be analysed together with a comprehensive priority degree assessment.

\section{Comprehensive priority-degree assessment}

The comprehensive priority-degree assessment is a priority-degree assessment that utilises the corresponding weight coefficients for the determination based on the

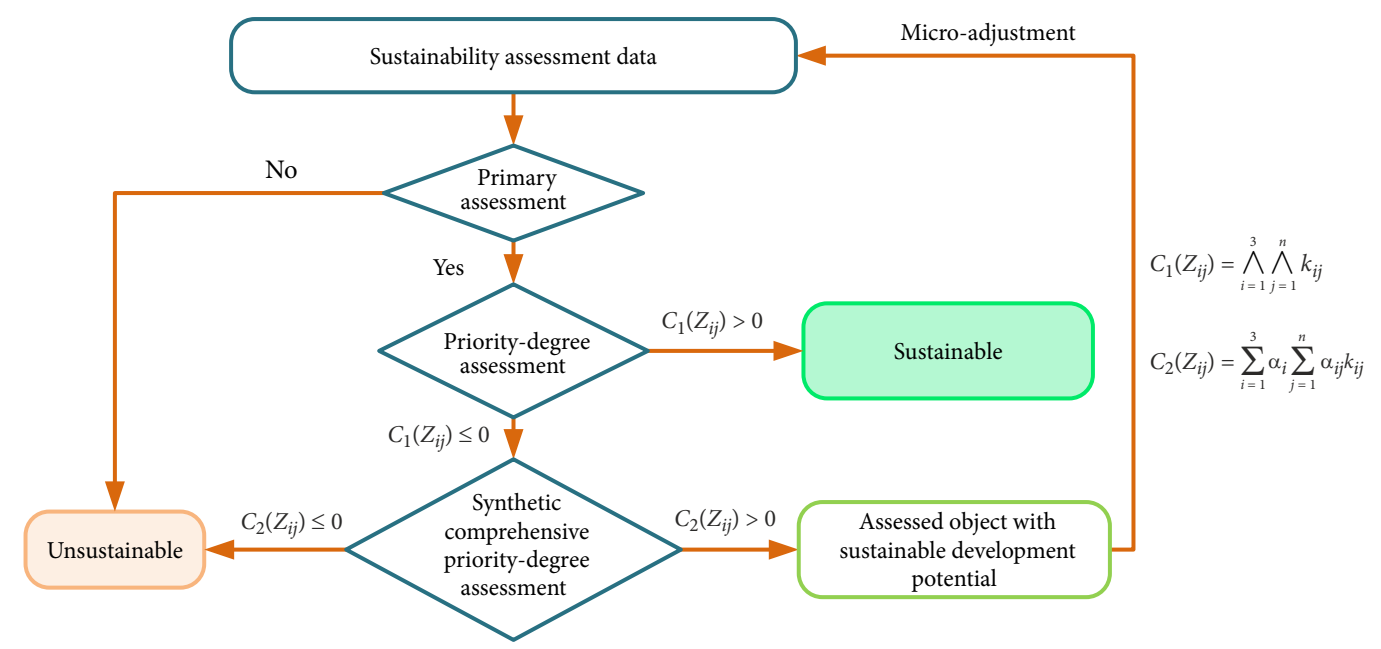

Figure 8. Assessment process based on extension priority degree assessment 
following formula:

$$
C_{2}\left(Z_{i j}\right)=\sum_{i=1}^{3} \alpha_{i} \sum_{j=1}^{n} \alpha_{i j} k_{i j} \text {. }
$$

The calculation of $C_{2}\left(Z_{i j}\right)$ is similar to formula (8).

- $C_{2}\left(Z_{i j}\right)>0$.

In this case, it is believed that the assessed object $Z$ has the potential for sustainable development based on the results of the comprehensive priority-degree assessment. In an actual project, combined with the indicators with a negative correlation degree, the project can be partially adjusted, which will generally allow it to pass the prioritydegree assessment. Moreover, to rate sustainability levels can enable stakeholders to compare the sustainability performance of reused industrial buildings in a standardised manner. This study adopts an approach similar to ASGB and other research (Ameen \& Mourshed, 2019) to divide the sustainability state into 4 levels, which are low, moderate, high, and very high, respectively. The reused industrial building is not awarded any star if the comprehensive value is below 0.4 at the first level. If the value is between 0.4 and 0.6 , the building can be awarded one star at the second level. The building can be awarded a two-star rate with a value between 0.6 and 0.8 at the third level. Finally, if the value is above 0.8 , the building can be awarded a three-star.

$$
\text { - } C_{2}\left(Z_{i j}\right) \leq 0 \text {. }
$$

This indicates that both assessments failed, and the assessed object did not have sustainable development potential. At this point, by comparing and analysing the normative correlation degrees of the assessment indicators, it can be found that there are many negative correlation degrees. In other words, the project needs major adjustments to pass the priority-degree assessment.

\section{Case study}

\subsection{Project overview}

This study considered a reuse project at Huaqing College, for which relatively complete basic data were available (Figure 9). Huaqing College involved a typical combination of reuse modes, which could be divided into education parks, creative parks, and residential areas. Based on this project, a sustainability assessment model was established for application analysis. Two methods were adopted to obtain the qualitative indicator value. In one method, basic data from the project were provided to the relevant experts, along with assessment values from experts. The other method was to find the participants in the implementation process of the project, and let them provide assessment values based on their construction experience.

\subsection{Data collection}

The basic data required for this case study were collected in the following ways.

\section{Basic information}

In co-operation with Huaqing College, information about the reuse process of this project was collected, along with information on all the economic indicators and some social and environmental indicators. This information provided reliable data for the assessment.

\section{Government report}

Information on indicators such as the 'ability to provide services' could be obtained by browsing through the statistics published by the government of the region where the project was located.

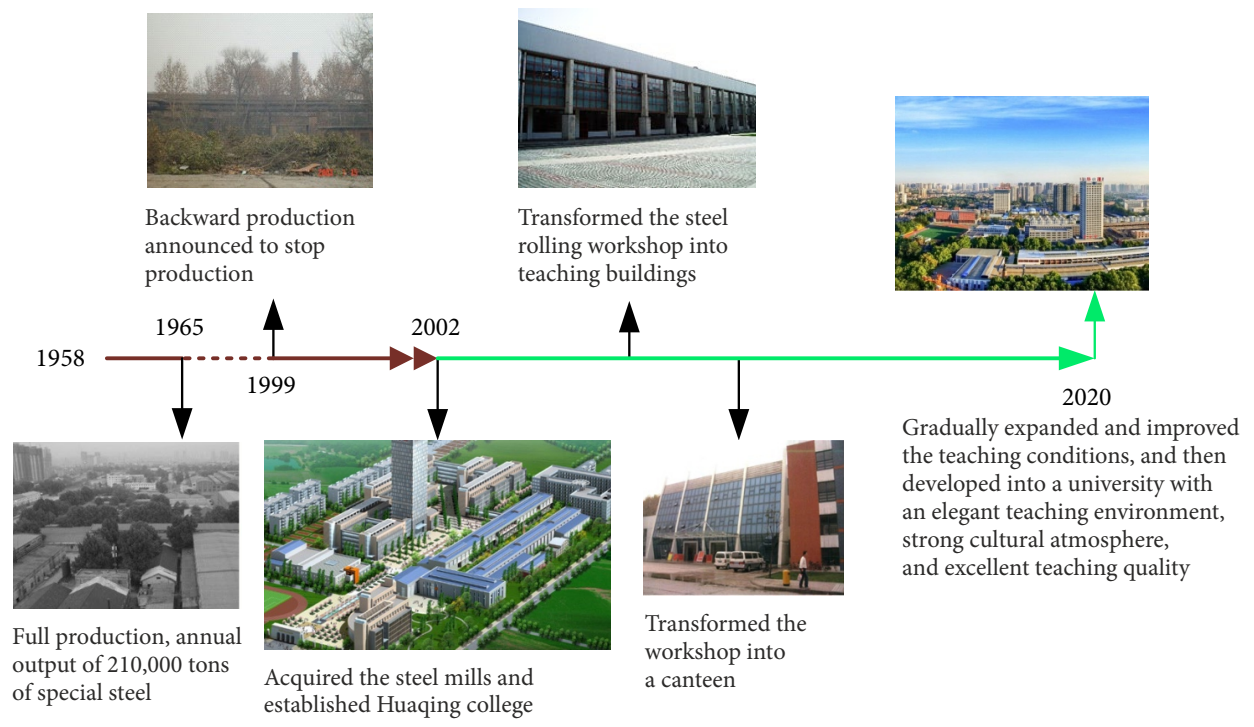

Figure 9. Reuse process for Huaqing College 


\section{Project survey}

A survey provided information to supplement the basic indicators obtained in the first two steps. This also made it possible to check the authenticity of the project information provided by project decision-makers and experts or collected by the website. If the data from different respondents were inconsistent, the investigators first had to verify it through an actual investigation and evidence collection. Afterwards, if there was a slight deviation in opinions, the investigators adhered to the opinions of the direct participants in the project and relevant experts.

\subsection{Result of assessment}

Based on the basic data collected for the reuse project, along with information from assessment experts and participants, the sustainability assessment of the reuse project utilised the following processes.

\section{Primary assessment}

This process mainly involved evaluating the construction materials of the reuse project and asking the construction personnel to verify that no illegal operation occurred during the project implementation process, the structural reinforcement met the reliability requirements, the structure was safe, and there were no major hazards during the operation. Because the assessment results indicated that the project met the mandatory requirements, the correlation degree could be calculated.

\section{Priority-degree assessment}

The normative correlation degree of each indicator was calculated according to the corresponding correlation function, and the results are listed in Table 1. A small value, $C_{1}\left(Z_{i j}\right)=\underset{i=1}{\stackrel{3}{\wedge}} \hat{j}_{j=1}^{n} k_{i j}=0$, indicated that the sustainability of the project could not be determined, and a comprehensive priority-degree assessment of the project needed to be conducted.

\section{Comprehensive priority-degree assessment}

The comprehensive priority degree was calculated using the calculated values in Table 1. For the missing indicators, the weight coefficients were equally assigned to other indicators.

$$
\begin{aligned}
& C_{2}\left(S I_{1}\right)=a_{11} \times K\left(S I_{11}\right)+a_{12} \times K\left(S I_{12}\right)+a_{13} \times K\left(S I_{13}\right)+ \\
& a_{14} \times K\left(S I_{14}\right)+a_{15} \times K\left(S I_{15}\right)+a_{16} \times K\left(S I_{16}\right)=0.6760 ; \\
& C_{2}\left(S I_{2}\right)=\alpha_{21} \times K\left(S I_{21}\right)+\alpha_{22} \times K\left(S I_{22}\right)+\cdots+ \\
& \alpha_{28} \times K\left(S I_{28}\right)=0.72 ; \\
& C_{2}\left(S I_{3}\right)=\alpha_{31} \times K\left(S I_{31}\right)+\alpha_{32} \times K\left(S I_{32}\right)+\cdots+ \\
& \alpha_{312} \times K\left(S I_{312}\right)=0.5993 ; \\
& C_{2}(S I)=\alpha_{1} \times C\left(S I_{1}\right)+\alpha_{2} \times C\left(S I_{2}\right)+\alpha_{3} \times C\left(S I_{3}\right)=0.6532 .
\end{aligned}
$$

According to the calculated results, the project passed the comprehensive priority-degree assessment but did not pass the priority-degree assessment, indicating that the project was in a state of sustainable development on the whole, but needed to be adjusted locally. The prioritydegree assessment result of indicator $S_{34}$ was zero. This indicated that the energy-saving measures adopted in the reuse project provided a small reduction in energy consumption but did not meet the relevant provisions. Overall, the sustainability performance of Huaqing College was 'high', which indicated the college was a relatively balanced and sustainable reuse project in terms of the economy, society, and environment and could be awarded a two-star. Also, our research group conducted sustainability assessments on other projects with complete survey information in the database and gave corresponding sustainability ratings.

\section{Discussion}

An indicator correlation radar diagram was drawn based on the results, as shown in Figure 10. The sustainability performances of the three factors were ranked from high to low: society (0.72), economy (0.6778), and environment (0.5993). The overall good social performance of the project was consistent with the satisfaction of the respondents. During the survey, the interviewed users (teachers, students, and tenants) and surrounding residents gave high praise to the reuse of old industrial buildings in Huaqing College. The assessment values of the economic indicators were balanced, and the economic performance of the project was good, which was better than other local reuse projects. The environmental assessment value was relatively low. This was mainly to balance the relationship between environmental and economic sustainability. A large amount of the cost of the reuse project came from environmental restoration. However, even though the environmental assessment value was the lowest, it still had good sustainability. After the operation, the project was awarded the title of a green building demonstration base by the local government.

\section{Economic factors}

The project had good economic sustainability, mainly because of its investment plan $\left(S I_{11}\right)$, the combination of three reuse modes tailored to local conditions, and the use of low-cost reuse techniques $\left(S I_{13}\right)$, through which the project made full use of the original building materials to reduce costs. Moreover, the financing model of the project showed diversity, including government subsidies and social capital, which guaranteed sufficient funds. In addition, the reuse cost per square metre $\left(S I_{14}\right)$ of the project was relatively low, at only $60 \%$ of the local construction cost for new buildings, even though it was not the lowest local reuse cost. Because of reasonable investment decisions, the dynamic PP $\left(S I_{15}\right)$ and IRR $\left(S I_{16}\right)$ values of the project both satisfied the owner. A period of observation of similar local projects showed that this project was one of the few to achieve good economic returns, with most of the other projects suffering losses or going bankrupt. 
a) Economic indicators

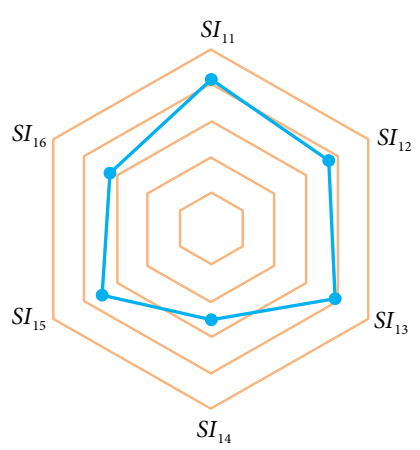

b) Social indicators

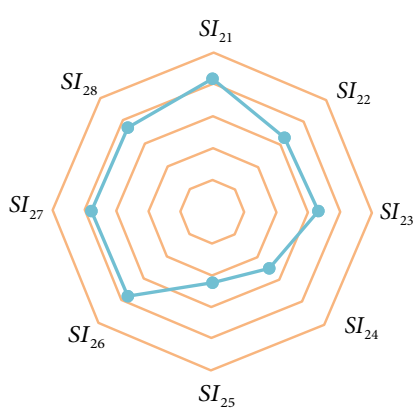

c) Environmental indicators

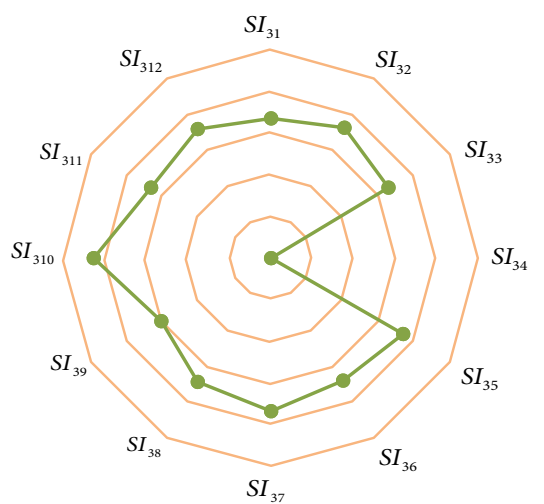

Figure 10. The indicator correlation degree

\section{Social factors}

In the later stage of the operation, the majority of the employees were the original factory workers, and the absorption of external labour decreased. On the other hand, to ensure campus security and the normalisation of teaching order, the college was less open and had limited ability to provide services to the region. Therefore, the indicators of the ability to provide employment $\left(S_{24}\right)$ and ability to provide services $\left(S I_{25}\right)$ had low scores. However, it solved the problem of the re-employment of the original factory employees and enhanced social influence. The reuse improved the office conditions and street images inside and outside the steel factory. In this way, the college was in harmony with the surrounding residents to a certain extent. In addition, in the process of reuse, the original industrial equipment and materials were adequately utilised, and the original appearance of the old industrial buildings was preserved to the greatest extent possible so that the industrial heritage and culture could be fully protected.

\section{Environmental factors}

Except for the prominent values of the reduction in energy consumption $\left(\mathrm{SI}_{34}\right)$ and noise pollution $\left(\mathrm{SI}_{310}\right)$ indicators, the values were between 0.6 and 0.8 . This showed the advantages of reuse, making full use of old industrial resources, and deploying low-cost technologies to remediate the environment according to local conditions to reduce reuse costs. Because the correlation value of indicator $S_{34}$ was zero and it had a large weight, the priority degree of the 'environmental indicator' was significantly lower than those of the other two items, making the overall assessment value lower. The project investigation and interviews of project construction personnel showed that many of the teaching buildings reclaimed from reused industrial buildings adopted non-thermal insulation glass curtain walls, which have poor thermal insulation performance and no obvious energy-saving effect. After the sustainability assessment, improvement measures were implemented, and the deficiencies of the follow-up projects in this respect were improved. In addition, because of the distance from the centre of the city and the teaching needs of the college, the noise impact during construction was small.
Compared with new construction projects, this project made reasonable use of the original buildings, roads, trees, and other resources of the steel factory, which greatly reduced the construction waste. The land and various types of building materials were saved, which directly increased the use of renewable materials.

\section{Assessment indicators and model}

Because there was no useful and effective sustainability assessment indicator system and assessment method for reused industrial buildings for project stakeholders in China, based on the literature analysis and surveys, an assessment indicator system was established based on the economy, society (including culture), and environment (Almeida et al., 2018). Based on the sustainability assessments of other projects (Ameen \& Mourshed, 2019) or similar projects (Tan et al., 2018a), the applicable sustainability assessment indicators for reused industrial buildings were determined through investigations, screening, and supplementation. The indicators were highly targeted at reused industrial buildings and could comprehensively reveal their sustainability problems (Fan et al., 2013). The indicator weights integrated subjective and objective factors, which made the assessed sustainability consistent with the actual situation. The multi-level assessment model was found to be more practical than other models. The priority-degree assessment could determine the sustainability issues for all of the aspects of reused industrial buildings and provided a basis for decision-making to improve sustainability. Furthermore, the comprehensive priority-degree assessment had a great impact on the sustainability rating and social reputation of the reused industrial building project.

In short, through the case study and comparative analysis, it can be seen that the established assessment indicator system could better reflect the characteristics of reused industrial buildings. Moreover, with the aid of the proposed assessment indicator weights and model, specific sustainability problems on reused industrial buildings in economic, social, and environmental dimensions could be found, and a reasonable sustainability rating could be given. 


\section{Conclusions}

There are currently a large number of idle industrial buildings that could be reused in China, which has been highly concerned by local governments and related developers. However, stakeholders have no effective sustainability assessment approach for reused industrial buildings to rate the sustainability performance of a project to promote the sustainable development of the project in many aspects.

To this end, this study analysed the factors influencing the sustainable development of reused industrial buildings through literature analysis, investigation on projects of reused industrial buildings, and a questionnaire survey, and applied factor analysis to group 26 assessment indicators into three dimensions: economy, society, and environment. These indicators could not only effectively reflect the influencing factors, but also met the assessment process. The goal of sustainable development sought by a reuse project is the economic-social-environmental balance. The indicator weights were assigned to balance these three aspects of the assessment process, and this reasonable weight assignment method was helpful in balancing the impacts of the indicators. The sustainability assessment extenics-based model, which offered the advantage of overcoming the incompatibility between linguistic variables and metric variables, was suitable for determining the sustainability performance of reused industrial buildings. In particular, the three types of correlation functions established according to the attributes of the indicators and the differences between their value ranges could further improve the applicability and effectiveness of the method.

The sustainability assessment extenics-based model was applied in a reuse project. The results showed that those 26 assessment indicators can fully and accurately display the sustainability deficiencies of the project. From a practical point of view, the results of the case study also validated the applicability and effectiveness of the assessment model. The priority-degree of each indicator can be provided for decision-makers or operators to determine the specific weaknesses of the buildings and identify the parts that need to be improved and maintained in order to improve the sustainability of the buildings. Meanwhile, sustainability rating agencies can apply this assessment system to assess the overall sustainability level of a project or award a sustainability rating for related governmental departments or developers. In addition, the findings can provide a reference for the formulation of future policies and sustainability standards on reused industrial buildings.

However, the diversity of the surveys made data collection more difficult, and experts from different specialities affected the indicator optimisation and weights. Different regions have great differences in their economic, social, and environmental requirements for sustainable development in China. The sustainability assessment did not involve regional differentiation because of the limited data. In future research, we will continue to focus on those projects on the reuse of old industrial buildings and col- lect their information to enlarge the preliminary database. Based on the enlarged database, the assessment indicator system will be further adjusted and extended, especially to supplement indicators on culture and policies. Moreover, the assessment method will be applied to a large number of projects to modify the indicator weights and model to improve its applicability and accuracy.

\section{Acknowledgements}

The authors sincerely thank Huaqing College for providing data support for this study.

\section{Funding}

This work was financially supported by the National Natural Science Foundation of China (Grant No. 51908452); Special Scientific Research Plan of Education Department of Shaanxi Province (Grant No. 18JK0470).

\section{Author contributions}

Tian Wei and Zhong Xingju researched, summarized literature. Goh Yang Miang and Tian Wei designed the methodology. Zhang Guangmin analysed the influenceing factors. All of the authors contributed to the setting of sustainability indicators. Tian Wei and Zhong Xingju finished the sustainability assessment model. Zhang Guangmin collected the data of HuaQing college, and Zhong Xingju performed calculations. All of the authors contributed to discussion of results and conclusions of the paper.

\section{References}

Ali, H. H., \& Al Nsairat, S. F. (2009). Developing a green building assessment tool for developing countries a case of Jordan. Building and Environment, 44, 1053-1064. https://doi.org/10.1016/j.buildenv.2008.07.015

Almeida, C. P., Ramos, A., \& Silva, J. A. (2018). Sustainability assessment of building rehabilitation actions in old urban centres. Sustainable Cities and Society, 36, 378-385.

https://doi.org/10.1016/j.scs.2017.10.014

Ameen, R. F. M., \& Mourshed, M. (2019). Urban sustainability assessment framework development: The ranking and weighting of sustainability indicators using analytic hierarchy process. Sustainable Cities and Society, 44, 356-366.

https://doi.org/10.1016/j.scs.2018.10.020

Atanda, J. O. (2019). Developing a social sustainability assessment framework. Sustainable Cities and Society, 44, 237-252. https://doi.org/10.1016/j.scs.2018.09.023

Banani, R., Vahdati, M. M., Shahrestani, M., \& ClementsCroome, D. (2016). The development of building assessment criteria framework for sustainable non-residential buildings in Saudi Arabia. Sustainable Cities and Society, 26, 289-305. https://doi.org/10.1016/j.scs.2016.07.007

Borwein, J. M., \& Zhu, Q. J. (2016). A variational approach to Lagrange multipliers. Journal of Optimization Theory and Applications, 171, 727-756.

https://doi.org/10.1007/s10957-015-0756-2 
Chan, A., Cheung, E., \& Wong, I. (2015a). Impacts of the Revitalizing Industrial Buildings (RIB) scheme in Hong Kong. Sustainable Cities and Society, 19, 184-190.

https://doi.org/10.1016/j.scs.2015.08.005

Chan, A., Cheung, E., \& Wong, I. (2015b). Revitalizing industrial buildings in Hong Kong - A case review. Sustainable Cities and Society, 15, 57-63.

https://doi.org/10.1016/j.scs.2014.10.004

Chan, A., Cheung, E., \& Wong, I. (2015c). Recommended measures on the revitalizing industrial buildings scheme in Hong Kong. Sustainable Cities and Society, 17, 46-55. https://doi.org/10.1016/j.scs.2015.03.012

Drejeris, R., \& Kavolynas, A. (2014). Multi-criteria assessment of building sustainability behavior. Procedia - Social and Behavioral Sciences, 110, 502-511.

https://doi.org/10.1016/j.sbspro.2013.12.894

Eom, J. S., \& An, D. W. (2018). Regeneration of industrial facilities into cultural facilities in Seoul: Studying location value. Sustainability, 10, 47-78. https://doi.org/10.3390/su10124778

Fan, S. J., Sheng, J. X., Li, H. M., \& Yan, R. Q. (2013). Potential assessment of old industrial buildings reuse utilization based on combination weight method. Industrial Construction, $10,002$.

Gu, P. F., Xi, W., Ye, W. P., Shi, J., \& Zhao, J. (2019). Extenics matter-element analysis on dilemma problem in HMI design of nuclear power plant. Nuclear Engineering and Design, 350, 176-181. https://doi.org/10.1016/j.nucengdes.2019.05.014

Hannouf, M., \& Assefa, G. (2018). A life cycle sustainability assessment-based decision-analysis framework. Sustainability, 10, 3863. https://doi.org/10.3390/su10113863

He, H., Liu, W., Yu, J., \& Li, X. (2016). Extenics-based testing method of divergent thinking quotient. Procedia Computer Science, 91, 151-157.

https://doi.org/10.1016/j.procs.2016.07.052

Jiang, P., Li, Ch., Li, R. R., \& Yang, H. F. (2019). An innovative hybrid air pollution early-warning system based on pollutants forecasting and Extenics evaluation. Knowledge-Based Systems, 164, 174-192.

https://doi.org/10.1016/j.knosys.2018.10.036

Lee, J. H., \& Lim, S. (2018). An Analytic Hierarchy Process (AHP) approach for sustainable assessment of economybased and community-based urban regeneration: The case of South Korea. Sustainability, 10, 4456.

https://doi.org/10.3390/su10124456

Li, H. M., Tian, W., \& Yan, R. Q. (2013). Constituting recycling assessment indicator system for old industrial buildings (group). Xian University of Architecture \& Technology (Natural Science Edition).

Li, W. J., Song, Z. H., Mao, E. \& Suh, S. (2018). Using Extenics to describe coupled solutions in Axiomatic design. Journal of Engineering Design, 30, 1-31.

https://doi.org/10.1080/09544828.2018.1550633

Liu, Q. Y., Wang, M. W., Zhou, T. L., Shen, F. Q., \& Jin, J. L. (2019). A connection cloud model coupled with extenics for water eutrophication evaluation. Earth Science Informatics, 12, 659-669. https://doi.org/10.1007/s12145-019-00403-1

Lou, J. J., Gui, F. Z., Ren, S. D., Xie, Z. W., \& Zhao, Y. W. (2018). Improved TRIZ based on extension innovation method. Computer Integrated Manufacturing Systems, 1, 13.

Mahmoud, S., Zayed, T., \& Fahmy, M. (2019). Development of sustainability assessment tool for existing buildings. Sustainable Cities and Society, 44, 99-119.

https://doi.org/10.1016/j.scs.2018.09.024
Mu, E. \& Pereyra-Rojas, M. (2017). Practical decision making An introduction to the Analytic Hierarchy Process (AHP) using Super Decisions. Springer.

https://doi.org/10.1007/978-3-319-68369-0_2

Nilashi, M., Zakaria, R., Ibrahim, O., Majid, M. Z. A., Zin, R. M., Chugtai, M. W., Abidin, N. I. Z., Sahamir, S. R., \& Yakubu, D. A. (2015). A knowledge-based expert system for assessing the performance level of green buildings. Knowledge-Based Systems, 86, 194-209.

https://doi.org/10.1016/j.knosys.2015.06.009

Peng, D.-g., Wei, T., Zhao, H.-r., Yao, J., \& Wang, W.-j. (2019). Cyber security risk assessment of power plant control system based on D-AHP and TOPSIS. Control and Decision, 11, 0240 .

Polat, G. (2016). Subcontractor selection using the integration of the AHP and PROMETHEE methods. Journal of Civil Engineering and Management, 22(8), 1042-1054.

https://doi.org/10.3846/13923730.2014.948910

Qin, X., Li, H.Q., \& Mo, Y. Y. (2017) Study on establishment and evaluation of risk network in green building projects based on SNA. China Civil Engineering Journal, 50(2), 119-131.

Rani, P., Mishra, A. R., Mardani, A., Cavallaro, F., Alrasheedi, M., \& Alrashidi, A. (2020). A novel approach to extended fuzzy TOPSIS based on new divergence measures for renewable energy sources selection. Journal of Cleaner Production, 257, 120352. https://doi.org/10.1016/j.jclepro.2020.120352

Saraiva, T. S., De Almeida, M., Bragança, L. \& Barbosa, M. T. (2018). Environmental comfort indicators for school buildings in sustainability assessment tools. Sustainability, 10(6), 1849. https://doi.org/10.3390/su10061849

Si, J., Marjanovic-Halburd, L., Nasiri, F., \& \& Bell, S. (2016). Assessment of building-integrated green technologies: A review and case study on applications of Multi-Criteria Decision Making (MCDM) method. Sustainable Cities and Society, 27, 106-115. https://doi.org/10.1016/j.scs.2016.06.013

Tan, R. R., Avis, K. B., Huelgas, A. P., \& Promentilla, M. A. B. (2014). Fuzzy AHP approach to selection problems in process engineering involving quantitative and qualitative aspects Process. Safety and Environmental Protection, 92, 467-475. https://doi.org/10.1016/j.psep.2013.11.005

Tan, Y. T., Shuai, C. Y., \& Wang, T. (2018a). Critical success factors (CSFs) for the adaptive reuse of industrial buildings in Hong Kong. International Journal of Environmental Research and Public Health, 15(7), 1546.

https://doi.org/10.3390/ijerph15071546

Tan, Y. T., Liu, G., Zhang, Y., Shuai, C. Y., \& Shen, Q. P. (2018b). Green retrofit of aged residential buildings in Hong Kong: A preliminary study. Building and Environment, 143, 89-98. https://doi.org/10.1016/j.buildenv.2018.06.058

Tokbolat, S., Karaca, F., Durdyev, S., Nazipov, F., \& Aidyngaliyev, I. (2018). Assessment of green practices in residential buildings: A survey-based empirical study of residents in Kazakhstan. Sustainability, 10, 4383.

https://doi.org/10.3390/su10124383

Tupenaite, L., Kaklauskas, A., Lill, I., Geipele, I., Naimaviciene, J., Kanapeckiene, L., \& Kauskale, L. (2018). Sustainability assessment of the new residential projects in the Baltic States: A multiple criteria approach. Sustainability, 10, 1387. https://doi.org/10.3390/su10051387

Tupenaite, L., Lill, I., Geipele, I., \& Naimaviciene, J. (2017). Ranking of sustainability indicators for assessment of the new housing development projects: Case of the Baltic States. Resources, 6, 55. https://doi.org/10.3390/resources6040055 
Turcu, C. (2013). Re-thinking sustainability indicators: Local perspectives of urban sustainability. Journal of Environmental Planning Management, 56, 695-719. https://doi.org/10.1080/09640568.2012.698984

Vucicevic, B., Stojiljkovic, M., Afgan, N., Turanjanin, V., Jovanovic, M., \& Bakic, V. (2013). Sustainability assessment of residential buildings by non-linear normalization procedure. Energy and Buildings, 58, 348-354. https://doi.org/10.1016/j.enbuild.2012.10.012

Wang, B., Li, H. N., Yuan, X. C., \& Sun, Z. M. (2017). Energy poverty in China: a dynamic analysis based on a hybrid panel data decision model. Energy, 10(12), 1942. https://doi.org/10.3390/en10121942

Zhang, X., Wu, Y., Shen, L., \& Skitmore, M. (2014). A prototype system dynamic model for assessing the sustainability of construction projects. International Journal of Project Management, 32, 66-76.

https://doi.org/10.1016/j.ijproman.2013.01.009

Zhang, L. M., Geng, Y., Dong, H., Zhong, Y., Fujita, T., Xue, B., \& Park, H.-s. (2016). Emergy-based assessment on the brownfield redevelopment of one old industrial area: a case of Tiexi in China. Journal of Cleaner Production, 114, 150-159. https://doi.org/10.1016/j.jclepro.2015.05.065

Zhang, H. D., Zhang, T. Y., Li, T., \& Zhang, T. H. (2018). Forecast of air quality pollutants' concentrations based on BP neural network multi-model ensemble method. China Environmental Science, 38(4), 1243-1256.

Zhou, Y., Hao, L., \& Liu,W. (2016). Extenics-based study on evaluation of urban community home-care service for the elderly. Procedia Computer Science, 91, 576-580.

https://doi.org/10.1016/j.procs.2016.07.146 